\title{
Generation of episodic magnetically driven plasma jets in a radial foil $\mathrm{Z}$-pinch
}

Francisco Suzuki-Vidal, Sergey V. Lebedev, Simon N. Bland, Gareth N. Hall, George

Swadling, Adam J. Harvey-Thompson, Jeremy P. Chittenden, and Alberto Marocchino Imperial College London, Blackett Laboratory, Prince Consort Road, London SW' 2BW, United Kingdom.

\author{
Andrea Ciardi \\ LERMA, Université Pierre et Marie Curie, Observatoire de Paris and École Normale Supérieure. UMR 8112 CNRS.
}

Adam Frank, Eric G. Blackman

University of Rochester, Department of Physics and Astronomy, Rochester, NY, USA.

Simon C. Bott

Center for Energy Research, University of California, San Diego, CA, USA.

(Dated: September 30, 2010)

\begin{abstract}
We present experimental results of the formation of magnetically driven plasma jets, showing for the first time a way of producing episodic jet/ouflows in the laboratory. The jets are produced using a $6.5 \mu \mathrm{m}$ thick aluminum disc (a radial foil), which is subjected to the $1 \mathrm{MA}, 250 \mathrm{~ns}$ current pulse from the MAGPIE generator [I. H. Mitchell et al., Rev. Sci. Instrum. 67, 1533 (1996)]. The early time motion of the foil is characterized by the bulk motion of the mass due to the magnetic pressure, together with the formation of a surface plasma following the direction of the $J \times B$ force. A low density plasma fills the region above the foil preceding the formation of subsequent magnetically driven jets on the axis of expanding magnetic bubbles. The outflows emerge in timescales of $\sim 30-40 \mathrm{~ns}$ and their episodic nature is the result of current reconnection in the foil, aided by the formation of current-driven instabilities in the jet and the distribution of mass available from the foil. The additional inductance due to the new current path inside the cavities was measured using an inductive probe, allowing to estimate the energy balance associated with the episodes. The measured temperature of the compressed jet resulted in $T_{e} \sim 300 \mathrm{eV}$ and a magnetic Reynolds number of $R e_{M} \sim 200-1000$, allowing the experiments to be in the regime relevant for scaled representations of astrophysical outflows.
\end{abstract}

PACS numbers: Valid PACS appear here

\section{INTRODUCTION}

The scaled study of astrophysical phenomena in laboratory experiments has seen major advances in recent years due to developments in the field of High-EnergyDensity Physics (HEDP). The generation of extreme states of matter involving high temperatures $(1-10 \mathrm{keV})$, densities $\left(10^{-5}-10^{1} \mathrm{~g} / \mathrm{cm}^{3}\right)$, and velocities $($ few $\times 100$ $\mathrm{km} / \mathrm{s}$ ), provides either a direct link to the physics encountered in a variety of astrophysical objects, or a scaled representation of astrophysical dynamics, such as jets (see [1] for a review).

Collimated outflows (jets) are in fact associated with widely diverse astrophysical environments that exhibit many common features independent of the central source [2], spanning a wide range of spatial and temporal scales. Classical examples are the galactic-scale jets powered by supermassive black holes [3], those generated as the end-product of dying solar-type stars (planetary nebulae [4]) and jets produced during star formation $[5,6]$. In general, it is believed that the ejection of jets relies on the conversion of gravitational energy into a Poynting flux which powers the outflows. The standard magnetohydrodynamic (MHD) models of jet formation rely on differential rotation along a large scale poloidal magnetic field $B_{P}$ to generate a toroidal magnetic field component $B_{\phi}$ which accelerates and collimates a disk-wind [7]. Our experiments are designed to model the acceleration and collimation of astrophysical jets taking place under the condition $\left|B_{\phi}\right| \gg\left|B_{P}\right|$.

Astrophysical jets and outflows are described to a first approximation by ideal MHD and our experiments are designed to produce flows in this regime. Its applicability requires the dimensionless Reynolds $(R e)$, magnetic Reynolds $\left(R e_{M}\right)$, and Peclet $(P e)$ numbers to be much larger than unity $[8,9]$. This implies that the transport of momentum, magnetic fields, and thermal energy, respectively, occurs predominantly through advection with the flow. It is important to emphasize that astrophysical jets have typical values of these dimensionless numbers of the order of $\sim 10^{7}-10^{15}$, which are many orders of magnitude greater than those obtained not only in laboratory experiments, but also in global numerical simulations of jet formation $\left(\sim 10-10^{3}\right)$, which have been so far the sole means of investigating time-dependent behavior of multi-dimensional MHD jets. The experiments discussed here extend the range of the dimensionless parameters obtained in the global modeling of jets. Typical values 
obtained are $R e \sim 10^{5}-10^{6}, R e_{M} \sim 200-500, P e \sim 20-50$, the ratio of thermal to magnetic pressure $\beta \sim 10^{-3}-10^{3}$, and the jets are radiatively cooled, similar to protostellar jets.

Laboratory jet experiments have been performed on a number of HED facilities with high-power lasers and high-current pulsed power generators. These include hydrodynamic jet experiments, in both adiabatic [10-14] and radiatively cooled [15-18] regimes, and experiments relevant to the study of the interaction of protostellar jets with an ambient medium [19-21] far away from the jet source.

High-current pulsed-power generators can naturally produce plasma jets in which magnetic fields are dynamically significant making these experiments relevant to different models of jet formation in which a toroidal (azimuthal) magnetic field is responsible for the collimation of the jet at the launching point. In experiments with radial wire arrays [19, 22-24] highly-supersonic, superAlfvenic, radiatively-cooled plasma jets are produced, which are confined by a toroidal magnetic field on the axis of a magnetic cavity. As the cavity expands, the jet was observed to undergo strong current-driven instabilities which, however, do not destroy the jet but produce a collimated, heterogeneous ("clumpy") jet.

Recently the use of a radial metallic foil on a Z-pinch driver has shown the formation of episodic plasma jets [25-27], each episode presenting similar dynamics to the single-episode jets seen in radial wire array experiments [22]. These episodic outflows can address open astrophysical questions regarding the spatial and temporal variability observed in protostellar jets.

In this paper we present results of a more detailed experimental study of the formation of episodic magnetically driven plasma jets. The rest of the paper is arranged as follows. Section II describes the experimental setup and briefly summarizes the generation of episodic magnetically driven jets. Section III presents the experimental results, discussing the early-time formation of plasma from the foil and its motion, the dynamics of episodic jets, their formation mechanism, the effect of instabilities, and estimates of temperature and of trapped magnetic field. Section IV summarizes our experimental results.

\section{EXPERIMENTAL SETUP}

The experimental setup (Fig. 1) is similar to the radial wire array configuration used in our previous jet experiments [22]. The main difference is that radial wires are now replaced by a thin radial metallic foil. An aluminium foil with a diameter of $\sim 55 \mathrm{~mm}$ and a thickness of $6-6.5$ $\mu \mathrm{m}$ is held in place between two concentric electrodes. The holder allows sufficient tension to be applied to the foil such that a flat surface is obtained. The central electrode (cathode) is a hollow stainless steel cylinder with a diameter of $\phi_{c}=3.1 \mathrm{~mm}$ in contact with the foil at its centre, pushing it axially $\sim 1 \mathrm{~mm}$ to ensure good elec-
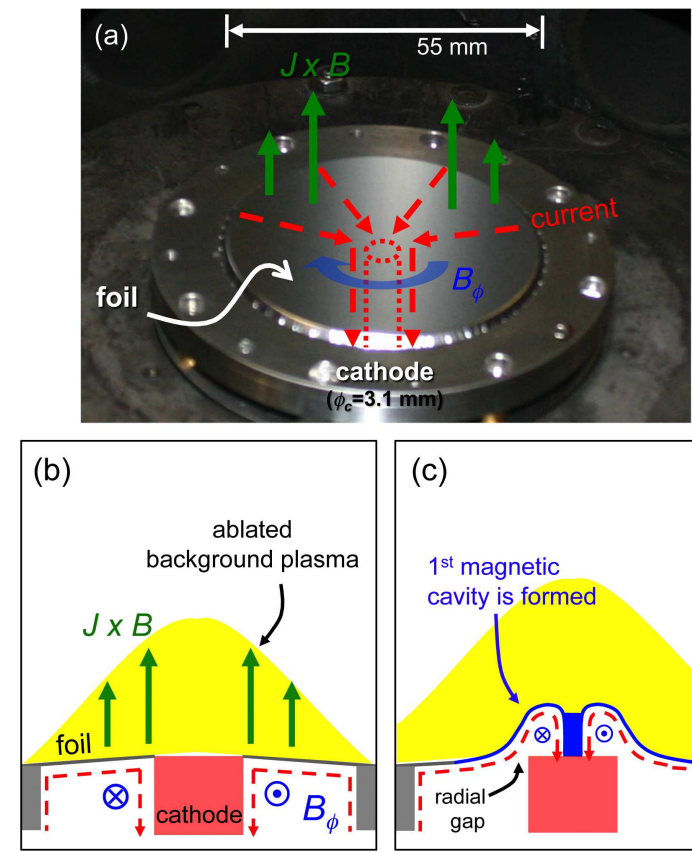

(c)

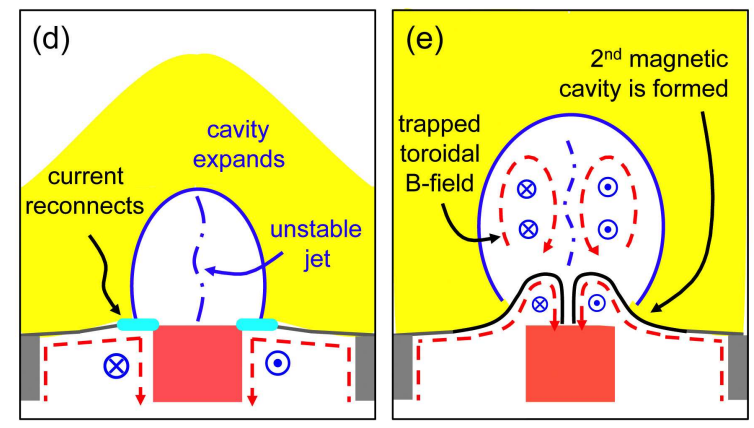

FIG. 1. (Color online) (a) A radial foil inside the discharge chamber of MAGPIE, with the cathode shown schematically (not to scale). (b)-(e) Schematic evolution of the foil showing the mechanism of episodic magnetic cavity formation triggered by current reconnection at the base of the cathode. Both figures show the current path (red-dashed arrows), toroidal magnetic field (blue arrows) and the resultant $J \times B$ force (green arrows).

trical contact with the foil during the experiment. The foil is driven by the $1 \mathrm{MA}, 250 \mathrm{~ns}$ current pulse from the MAGPIE generator [28], which can be well approximated as a $\sin ^{2}$ function (more details in Sec. IIIA). Fig. 1a shows a photograph of the radial foil placed inside the discharge chamber. This figure indicates the position of the cathode, the current path, the direction of the toroidal magnetic field $B_{\phi}$ and the resultant Lorentz $J \times B$ force, which is normal to the surface of the foil. The geometry of this configuration results in a current path along the central cathode, radially along the foil and along a return-current structure (the anode). The global toroidal magnetic field in the region below the foil is a function of the total current $I(t)$ and decreases with the radial distance from the axis $r$ as $B_{\phi}(r, t)=\frac{\mu_{0} I(t)}{2 \pi r}$. At peak current the toroidal magnetic field is $B_{\phi} \sim 100 \mathrm{~T}$ 


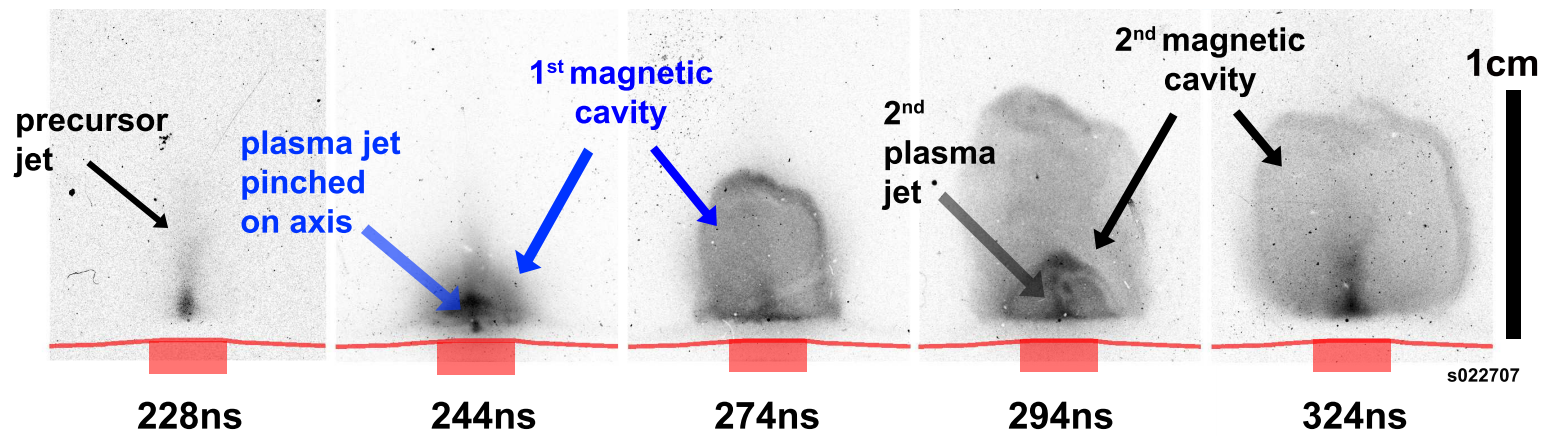

FIG. 2. (Color online) Side-on XUV emission from a radial foil showing its overall dynamics up to $\sim 320 \mathrm{~ns}$ (shot s022707). The initial position of the foil and the cathode (with a diameter of $3.1 \mathrm{~mm}$ ) are shown schematically.

(=1 MG) at the cathode radius.

Figs. 1b-e schematically illustrate the formation of a magnetically driven jet/outflow from a radial foil. The first two stages, plasma ablation and formation of the first magnetic cavity (Figs. 1b-c), are very similar (almost identical) to the formation of a magnetically driven jet in a radial wire array $[22,23]$. Two outflow components are generally present: a magnetic bubble (or cavity) accelerated by gradients of the magnetic pressure and surrounded by a shell of swept-up ambient material, and a magnetically confined jet in the interior of the bubble. The rate of expansion of the magnetic cavity itself depends on the density of the external medium. For the present experiments an ambient medium is provided by the plasma ablated from the foil at early times, before the formation of the cavity and the jet. Although the dynamics of the first magnetic bubble and jet are similar to those observed in radial wire array jets experiments, in the present work we are able to produce and observe for the first time the formation of several subsequent jets in a single experimental shot. There are two main differences between the radial foil and the radial wire array configurations, which could be responsible for the observed formation of multiple jet episodes in radial foil experiments. The first is the difference in the change of mass with radius (which is constant in radial wire arrays, but increases in a radial foil load). The second is the high initial azimuthal symmetry of the radial foil load (i.e. absence of gaps between the wires). Consequently, it is possible that the initial radial gap (Fig. 1c) produced by the full ablation of foil material at the cathode radius is smaller and can be more easily refilled by the plasma expanding from the foil and/or the cathode than in a radial wire array configuration. The gap closure allows the current to flow once again across the base of the magnetic cavity, thus re-establishing the initial configuration at this location. When the magnetic pressure is large enough to break through this newly deposited mass, a new jet/bubble ejection cycle begins.

The dynamics of the jet formation were studied with several plasma diagnostics: optical laser probing $(\lambda=532$ $\mathrm{nm}, \sim 0.4 \mathrm{~ns}$ exposure) was used for shadowgraphy, schlieren and interferometry; XUV self-emission $(h \nu>30$ $\mathrm{eV})$ from the plasma was recorded using multi-frame, time-resolved ( $\sim 3$ ns gate) pinhole imaging providing up to 8 frames per experiment; Photo-Conducting Detectors (PCDs) were used to measure the X-ray emission from the jets; magnetic "B-dot" probes measured any trapped magnetic field inside the outflows; an inductive probe connected to the cathode measured the voltage, thus allowing to calculate the Poynting flux driving the outflows; a spatially resolved, time integrated spectrometer utilising a spherically bent mica crystal measured the $\mathrm{X}$-ray spectrum, from which the electron temperature at different axial positions was estimated.

\section{EXPERIMENTAL RESULTS}

The XUV emission image in Fig. 2 illustrates the overall dynamics of the outflows formed in a radial foil configuration. At early times the ablated plasma expands axially away from the foil and forms a precursor jet on the axis. Details of the plasma evolution during this early stages are discussed in Sec. IIIA. This is followed by the formation several subsequent rapidly expanding magnetic cavities, which dynamics are presented in Sec. IIIB.

\section{A. Early-time foil motion and plasma formation}

As the current starts to flow through the foil, it is reasonable to expect that the magnetic pressure will be acting on all the mass if the current remains below the foil. It is possible then to use a 0-D approximation to calculate the expected initial motion of the foil (for a discussion of 0-D motion in Z-pinches see e.g. [29]). This is in contrast to wire arrays where the wire cores remain stationary until essentially all the mass is ablated, while at the same time the $J \times B$ force acts only on the ablated plasma which is free to flow around the wire cores, forming plasma streams perpendicular to the wires. It should be noted that the approximation discussed below is only valid for small axial displacements when the foil shape 


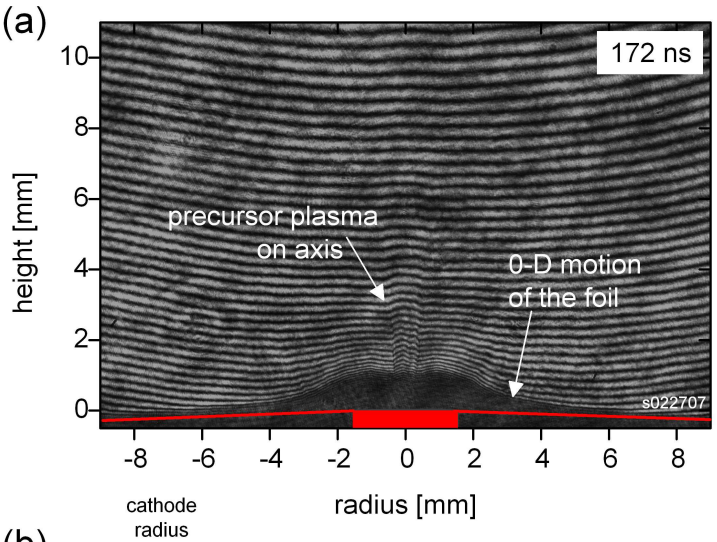

(b)

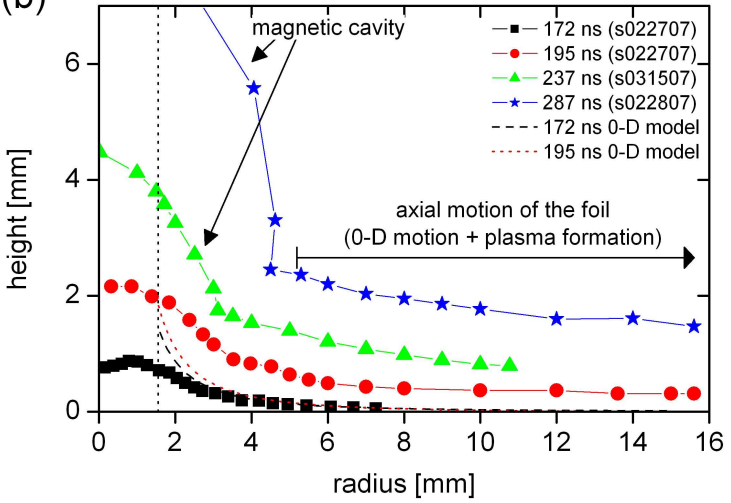

FIG. 3. (Color online) (a) Side-on laser interferogram at 172 ns showing the axial displacement of the foil as a dark region with respect to its initial position before the start of the current (shown schematically with the cathode). (b) Measured axial displacement of the foil from side-on laser probing at 172, 195, 237 and $287 \mathrm{~ns}$. Also shown are the calculated 0-D positions/displacements of the foil at 172 and $195 \mathrm{~ns}$, obtained by solving Eq. 1.

remains approximately planar and any current flowing in the axial direction, due to the axial displacement of the foil, can be neglected. By considering the magnetic pressure below the foil $P_{\text {mag }}(r)=\frac{B_{\phi}^{2}(r, t)}{2 \mu_{0}}$ acting on a infinitesimal ring with a radial length $\delta r$, for a foil with a thickness $\Delta$ and density $\rho$, the axial motion of the foil as a function of radial distance from the axis and time $z(r, t)$ is given by:

$$
\frac{d^{2} z(r, t)}{d t^{2}}=\frac{\mu_{0} I^{2}(t)}{8 \pi^{2} \rho r^{2} \Delta}
$$

Eq. 1 can be solved analytically by expressing the current from the MAGPIE generator as $I(t)=I_{\max } \sin ^{2}\left(\frac{\pi t}{2 t_{\max }}\right)$, where $I_{\max }$ is the peak current and $t_{\max }$ is the time of peak current.

The initial axial motion of the foil was measured from side-on laser probing images. A typical laser interferogram obtained at $172 \mathrm{~ns}$ is shown in Fig. 3a, where the bulk motion of the foil is seen as a dark, non-transparent region above the initial position of the foil marked on the image. The axial displacement increases towards smaller radius, which is consistent with a larger magnetic pressure closer to the cathode. Fig. 3b shows the evolution of this boundary measured from images taken at 172, 195, 237 and $267 \mathrm{~ns}$. The axial motion at $172 \mathrm{~ns}$ agrees well with the predicted 0-D motion from Eq. 1. At later times the observed axial displacement of the observed dark region is larger than that expected from the 0-D model. The discrepancy is in partly due to the breaking down of the assumption of small axial displacement, used to derive Eq. 1, but also because the dark boundary does not necessarily represent the position of the foil. This is especially clear from the observed large axial displacement occurring at large radii, where the $J \times B$ force is small and insufficient to provide such large axial motions. We interpret this as evidence of axial expansion of plasma from the foil, with sufficiently large electron density gradients $\left(\nabla n_{e}\right)$ to deflect the probing laser beam outside the optical system. The boundary of the dark region for the acceptance angle of our experimental setup then corresponds to $\int \nabla n_{e} d l \approx 2 \times 10^{20} \mathrm{~cm}^{-3}$, where $l$ is the path length along the plasma.

Above the boundary it is possible to measure the distribution of electron density of the plasma with laser interferometry, by comparing interferograms obtained during the experiment with a reference interferogram taken before the start of the current. Fig. 4a shows a 2-D map of line electron density $n_{e} l$ (integrated along the probing path, $\left.n_{e} l \equiv \int n_{e}(r, z) d l\right)$ obtained from the analysis of Fig. 3a with the IDEA interferometric software [30]. In this figure the contours represent regions of constant values of $n_{e} l$. It is seen that most of the plasma is concentrated close to the axis in the region above the cathode and decreases with radius, indicating a larger ablation rate of plasma at smaller radius, where the Lorentz $J \times B$ force is the strongest.

Radial profiles of $n_{e} l$ at different heights from the foil $(z=1,1.3,2,3$ and $3.5 \mathrm{~mm})$ are shown in Fig. $4 \mathrm{~b}$. Since the ablated plasma displays a good degree of azimuthal symmetry, the radial electron density distribution $n_{e}(r)$ can be obtained by applying Abel inversion to the radial profiles shown in Fig. 4b. Results of Abel inversion are shown in Fig. 4c. It is seen that the density distribution peaks at a radius approximately equal to the radius of the cathode, decreases for smaller radius, and peaks again on axis. The density profiles were numerically integrated in order to estimate the mass per unit radius, as a function of height, in the ablated plasma assuming a typical value for ionization of $Z=5$. The total mass of ablated plasma at the radius of the cathode (integrated between $1.55<r<1.57 \mathrm{~mm}$ ) constitutes $<1 \%$ of the initial mass from the foil from the same initial volume.

At the time of the image (172 ns) plasma is seen to expand to a maximum height of $\sim 6 \mathrm{~mm}$. Assuming that plasma is formed at the start of the current pulse this results in an axial expansion velocity of $V_{z} \sim 35 \mathrm{~km} / \mathrm{s}$. However it is most likely that plasma is formed not at $\mathrm{t}=0$ (current start) but at $\sim 50 \mathrm{~ns}$ when a flash of XUV 

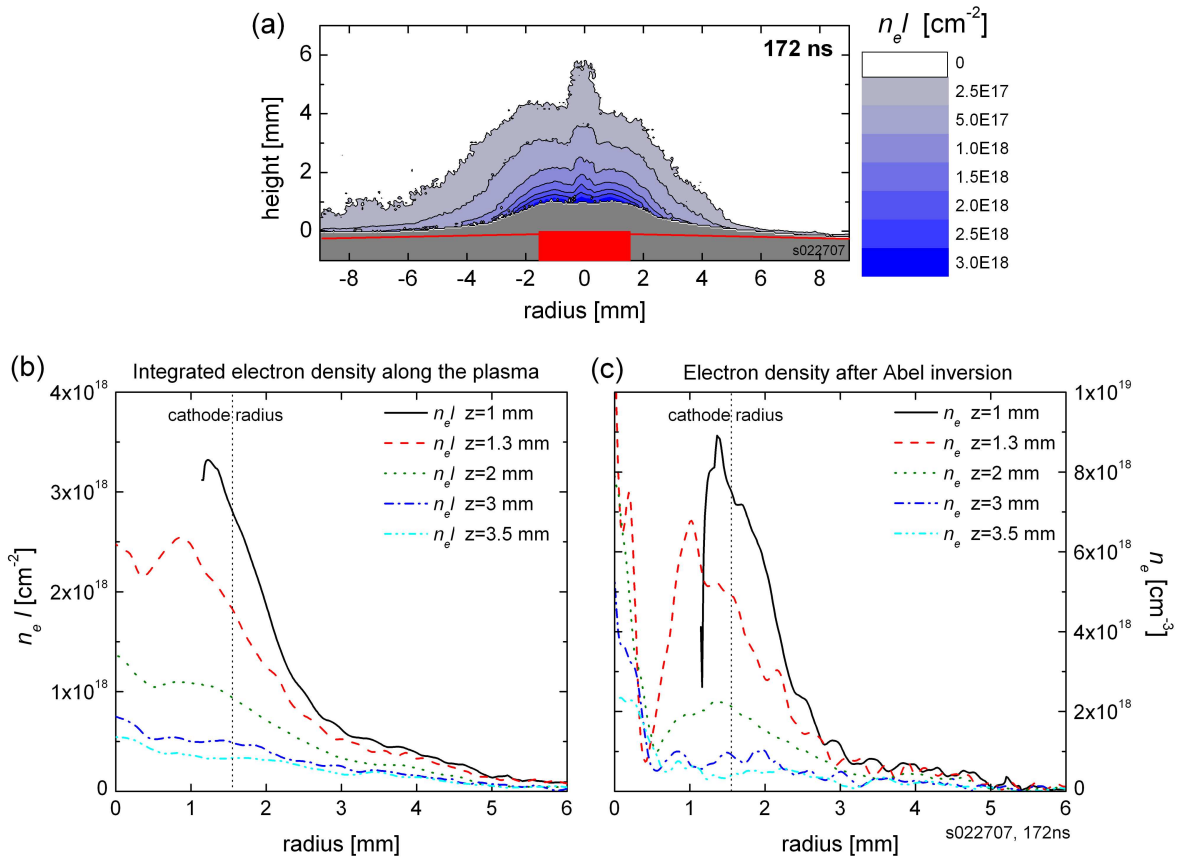

FIG. 4. (Color online) (a) 2-D map of electron density integrated along the plasma $\left(n_{e} l\right)$ at 172 ns obtained from Fig. 3a. (b)-(c) Radial profiles of $n_{e} l$ and Abel-inverted electron density profiles $\left(n_{e}\right)$, both at different heights from the foil. Data was obtained from the analysis of (a).

emission is detected from the surface of the foil. The time delay in the formation of plasma from the foil is similar to that observed in cylindrical wire arrays [31].

An interesting feature of the ablated plasma motion above the foil is the formation of a precursor plasma jet on the axis of the foil. This is seen in Fig. 4 as an increase of the electron density in the region above the cathode. As there is no ablation above the cathode, there should be an initially empty region on the axis, however plasma is redirected towards the axis by radial pressure gradients. It is also possible that some toroidal magnetic field (and current) are advected by the ablated plasma, and this magnetic field may also contribute to the acceleration of plasma toward the axis. The converging plasma flows are colliding on the axis forming a standing shock which redirects the flow in the vertical direction, forming a plasma jet in a similar way as in previous experiments where jets were produced using conical wire arrays [17]. This precursor jet can be used for studies of jet-ambient interactions if an ambient gas is introduced above the foil, and first results of such experiments are presented in [26].

Plasma formation in the region below the foil was also observed. Figs. 5a-b show side-on laser probing images taken at 247 and $270 \mathrm{~ns}$ respectively. These images indicate that only a small amount of plasma is expanding from the foil in a direction opposite to the $J \times B$ force. This was quantified using laser interferometry (Fig. 5a), resulting in values of $n_{e} l \lesssim 2.5 \times 10^{17} \mathrm{~cm}^{-2}$, which is significantly lower the measured values at the top side of the foil at the same times. Fig. 5 also shows that the formation of plasma in this region comes primarily from the surface of the cathode. This non-uniform plasma appears to be modulated along its length with an average wavelength of $\lambda=0.71 \pm 0.14 \mathrm{~mm}$, which is a factor of $\sim 3$ larger than the wavelength of the modulations of plasma observed on stainless steel cylindrical wire arrays $(\lambda \sim 0.2 \mathrm{~mm}[32])$. The images also show that the amount of plasma along the cathode reduces with increasing distance from the foil, which could indicate that XUV emission from the foil is affecting the formation of plasma on the cathode rod.

\section{B. Formation and dynamics of episodic jets}

Following the initial plasma formation $(\sim 200 \mathrm{~ns})$, the increasing drive current and Lorentz force lead to the largest rate of ablation and axial motion in the region at small radii close the cathode. Both the ablation and/or axial displacement of the mass in this region should lead to the formation of a radial gap between the cathode and the remaining foil. The formation of this radial gap is seen in numerical simulations of the experiment [25]. A magnetic bubble is then seen to rise through the ambient plasma, which is swept into a layer of shocked material surrounding the bubble. In the interior of the bubble the magnetic field present is able to confine a jet on axis, with the whole structure continually powered by the Poynting flux injected at the base of the cavity through the radial gap. At this stage the current flows along the jet and the cavity walls, which take up the magnetic stress of the 

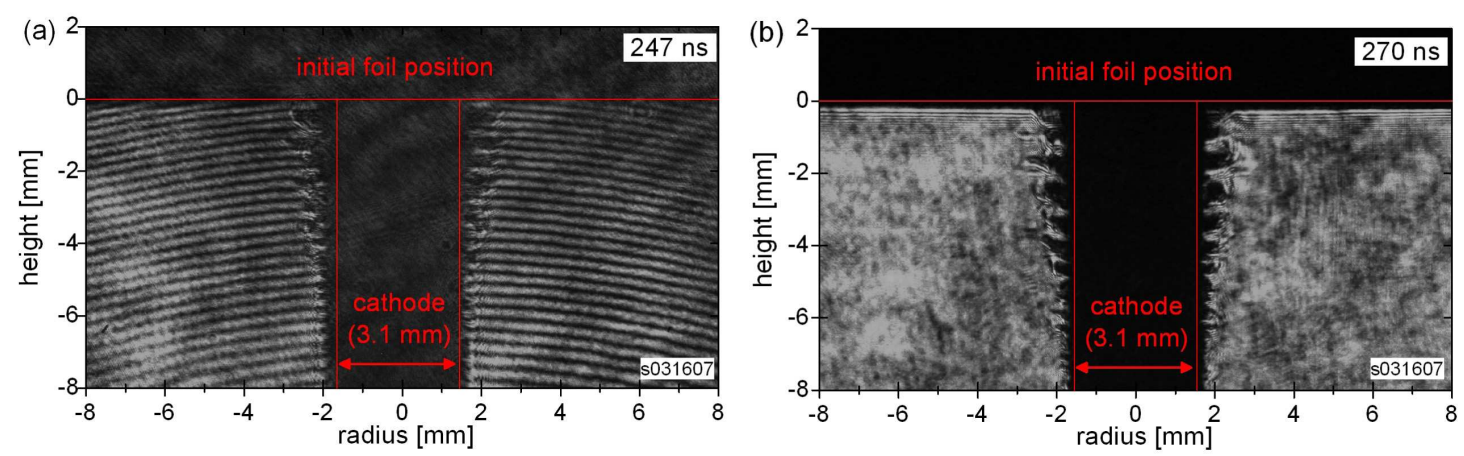

FIG. 5. (Color online) Side-on laser probing images of the region below the foil showing the formation of a non-uniform plasma on the surface of the cathode. (a) Laser interferogram at $247 \mathrm{~ns}$, (b) laser shadowgram at $270 \mathrm{~ns}$.

magnetic field present on the interior of the bubble and transfer it to the external medium, which is ultimately responsible for the collimation of the entire structure.

The most striking feature of these new experiments is the formation of several, subsequent outflows. This process can be clearly seen on XUV emission images (Fig. 2), obtained from the same experiment. The time sequence shows the formation of the precursor plasma jet on the axis (228 ns) followed by the formation and expansion of the first magnetic bubble (244 and $274 \mathrm{~ns}$ ). As the first bubble forms, $a \sim 2 \mathrm{~mm}$ long, highly-emitting plasma jet is seen on the axis. The emission from this compressed plasma is sufficiently energetic to be observed through the axially displaced foil, indicating that part of this process might be occurring below the foil. The formation of the second magnetic cavity is first seen on the image at 294 ns, with a clearly visible plasma jet on the axis. The presence of XUV emission from the walls of the magnetic cavities indicate that the plasma density and temperature are sufficiently high there; the wall of the cavity is essentially a shock wave driven through the ambient by the presence of the magnetic field.

Measurements of the temporal evolution of the maximum height and radius of the two subsequent magnetic cavities are shown in Figs. 6a-b respectively. The magnetic cavities expand with approximately constant velocity in both radial and axial directions. The axial velocities obtained from linear fits to the experimental data, for the first and second cavities, are $V_{Z 1}=146 \mathrm{~km} / \mathrm{s}$ and $V_{Z 2}=205 \mathrm{~km} / \mathrm{s}$ respectively. Their radial expansion velocities are a factor of $\sim 3$ slower, similar to previous experiments using radial wire arrays [22]. The increase in the overall expansion velocity of each new cavity was observed in all experiments and is consistent with the idea that earlier episodes sweep up the ambient medium, thus allowing new magnetic cavities to propagate through a lower density ambient plasma.

Figs. 6a-b also show the emission of soft X-rays (shown as red curves) measured with PCD detectors filtered with a $6 \mu \mathrm{m}$ polycarbonate filter (transmission of photons with energies between 200-300 eV and above $800 \mathrm{eV}$ ). The time of the X-ray emission is correlated with the time of formation of each cavity, as inferred from the extrapolation of the axial and radial trajectories back in time (marked with vertical arrows in Figs. 6a-b). This is an indication that each new episode starts with the pinching of plasma on the axis of the magnetic cavity and that pinched plasma is the source of the X-ray emission. The variation of intensity inside the second X-ray burst (starting at $\sim 280 \mathrm{~ns}$ ) might be related to the formation of current driven instabilities in the jet on the axis of the second cavity. Both the axial expansion dynamics and the periodicity of the X-ray emission show a timescale of $\sim 30-40 \mathrm{~ns}$ for the episodic formation of magnetic outflows.

The temporal evolution of the episodic jets and cavities at later times is presented in Fig. 7a, where the images were obtained during the same experiment. A succession of multiple cavities and embedded jets (for this particular shot 3-4 episodes) are seen propagating over length scales spanning more than an order of magnitude. The number of subsequent episodes can be inferred from the remnants of the cavity walls at their base, seen on the image at $406 \mathrm{~ns}$. The resulting flow is heterogeneous and clumpy, and it is injected into a long-lasting and well collimated channel made out of nested cavities. It is worth remarking that the bow-shaped envelope is driven by the magnetic field and not hydrodynamically by the jet, and measurements of the magnetic field inside the cavities are presented in Sec. III F.

\section{Estimate of an energy balance in the outflow}

The amount of energy delivered from the generator to the magnetic cavity and the jet can be estimated from the measurements of voltage at the cathode and the driving current. Using an inductive probe connected to the cathode, the voltage drop produced by the current path along the cathode, the jet, and the walls of the magnetic cavity was measured. The probe voltage $V_{i n d}$ is proportional to the time derivative of the magnetic flux produced by 

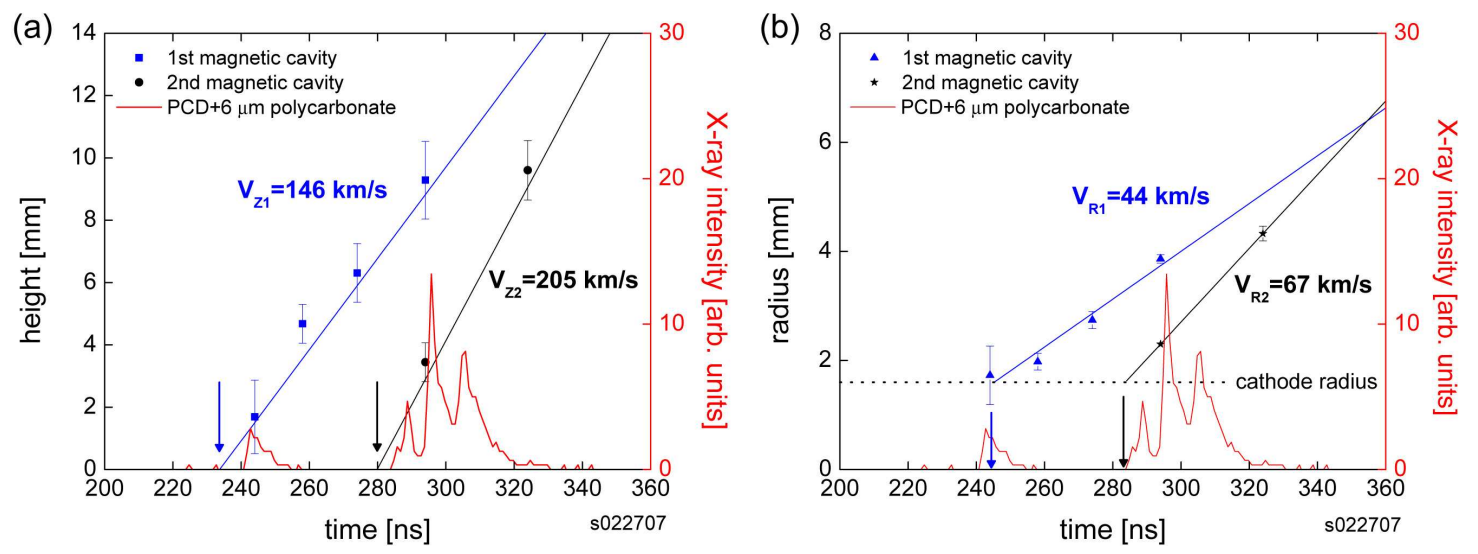

FIG. 6. (Color online) (a)-(b) Measured maximum height and radius of the two magnetic cavities shown in Fig. 2. The X-ray emission is also shown (in red).

this current $\left(V_{i n d}=\frac{d(L I)}{d t}\right)$, where $L$ is the inductance associated with the current path (with total current $I$ ). An example of these measurements is shown in Fig. 7b, where the voltage signal from a standard $6.5 \mu \mathrm{m}$ thick foil producing episodic outflows is compared with a reference shot, i.e. a foil with a thickness of $15 \mu \mathrm{m}$. In the case of the reference shot the foil did not move on the timescale of the experiment, the inductance was constant and the measured voltage was equal to $V_{i n d}=L \frac{d I}{d t}$.

For a $6.5 \mu \mathrm{m}$ thick foil the episodic jets were produced and the inductive probe shows rapid changes in voltage (seen at $\sim 220$ and $250 \mathrm{~ns}$ ) with respect to the voltage measurements from the reference foil. The timing of these voltage spikes agree with the X-ray emission bursts (shown at the bottom of Fig. 7b), which are in turn correlated to the formation of each jet/cavity. The deviations of the voltage from the reference case come from the additional (time-dependent) inductance linked to the jet and the magnetic bubble. Assuming that all current flows through the cavity, it is possible to find how the inductance changes in time. By taking the difference between the measured voltages from the $6.5 \mu \mathrm{m}$ and 15 $\mu \mathrm{m}$ shots and the known current, we estimate that the inductance of the cavity is $L \sim 1 \mathrm{nH}$. This is consistent with a geometrical estimate of the inductance from the size of the cavity at the same time, assuming the cavity can be approximated as two coaxial cylinders.

From this we can estimate the magnetic energy in the cavity to be $E_{M}=\frac{L I^{2}}{2} \sim 500 \mathrm{~J}$. It is also possible to estimate the total energy delivered to the cavity by the Poynting flux, by integrating $V_{i n d} I$, which gives $E_{P} \sim 600$ $800 \mathrm{~J}$. The difference between $E_{M}$ and $E_{P}$ will be shared between kinetic energy of the outflow, radiation and internal energy of the plasma. Further details on these measurements will be presented in future publications.

\section{Effect of instabilities on the dynamics of episodic magnetic cavities}

It is seen from Fig. 6a (e.g. at 294 ns) and Fig. 7a (e.g. at $346 \mathrm{~ns}$ and $406 \mathrm{~ns}$ ) that the jet on the axis of the magnetic cavity is unstable, breaking into a series of clumps along its length. This is similar to the situation discussed in our previous experiments with the generation of a single episode magnetically driven jet $[22,23]$. The development of instabilities and the subsequent disruption of the jet on the axis of the cavity could contribute to the increase of voltage at the base of the cavity and affect the process of reconnection of current through the gap between the cathode and the remnants of the foil, which is responsible for the formation of new jet episodes.

To investigate the possible effect of instabilities on the periodicity of the generation of magnetic cavities, a stainless-steel needle was placed on the axis of the system, normal to the surface of the foil. The needle was in contact with the foil, thus providing a fixed current path on the axis. The addition of the needle could delay the formation of MHD instabilities in the jet, which in turn are related to the process of current reconnection at the base of the cavities. Results from side-on laser probing and XUV emission images obtained from the same experiment are shown in Fig. 8a. The needle had an initial diameter of $200 \mu \mathrm{m}$ and a length of $10 \mathrm{~mm}$ and can be clearly seen on the image at 223 ns. The experimental data show that only a single magnetic cavity is formed in this case, with its dynamics qualitatively similar to the case of a foil without the needle on the axis. The plasma on axis remains localized preferentially along the length of the needle, becoming unstable after the cavity expands further along its tip, especially along the end of the needle.

Measurements of the height and radius of the cavity presented in Fig. 8b show that the cavity is forming at the same time as in the case without a needle. However, the axial and radial expansion velocities of the cavity are $V_{Z} \sim 340 \mathrm{~ns}$ and $V_{R} \sim 124 \mathrm{~km} / \mathrm{s}$ respectively, which are 
(a)

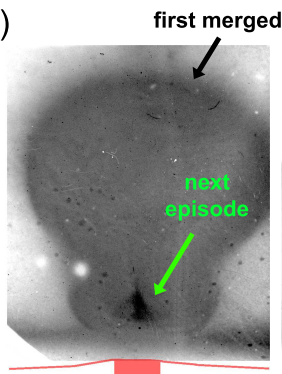

316ns

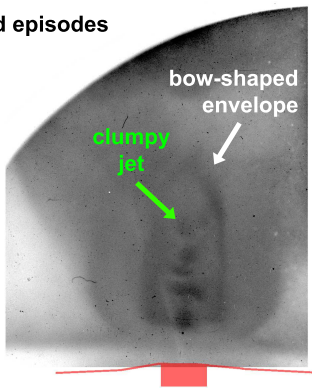

$346 n s$

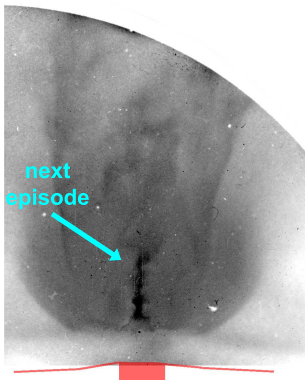

376ns

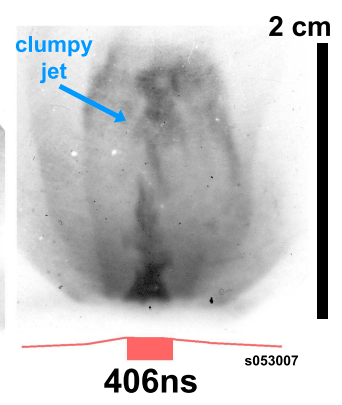

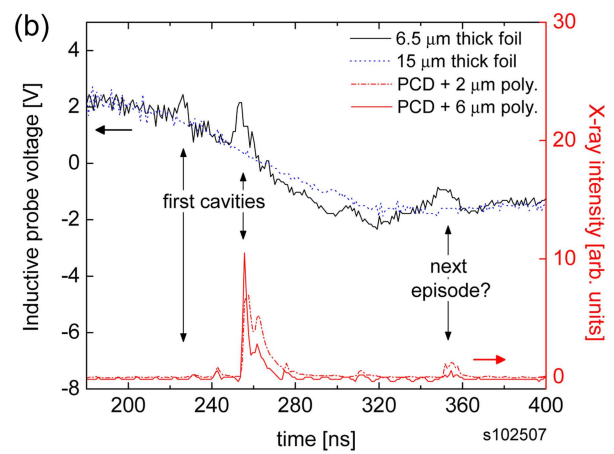

FIG. 7. (Color online) (a) Late-time evolution of the episodic magnetic cavities from 316 ns onwards (shot s053007). (b) Voltage signals from an inductive probe from a $6.5 \mu \mathrm{m}$ thick (solid black, shot s102507) and $15 \mu \mathrm{m}$ thick (dashed blue, shot s102407) foils. X-ray emission from a $6.5 \mu \mathrm{m}$ thick foil (red curves, shot s102507) from PCD detectors filtered with $2 \mu \mathrm{m}$ and $6 \mu \mathrm{m}$ thick polycarbonate. The X-ray emission is correlated to rapid changes in inductance due to the formation of episodic magnetic cavities.

40-50\% larger than without stabilization of the current with the needle (e.g. Fig. 6). The increase in the expansion velocity of the magnetic cavity suggests that a larger fraction of the current is localized inside the single magnetic cavity, increasing the magnetic pressure inside it and therefore the expansion velocities. Also shown in Fig. 8b is the X-ray emission during the experiment, which is characterized by two bursts. The first burst agrees with the time of formation of the magnetic cavity, whereas the second peak of emission occurs $\sim 30$ ns later, showing a higher intensity of X-ray emission in respect to the first one. The time of the second X-ray burst coincides with the time when the cavity reaches the end of the needle and could be related to the pinching or detachment of plasma above the needle. We cannot exclude the possibility of the formation of a second jet episode, though no second cavity is detected by laser probing or XUV emission diagnostics. More investigations are required here to clarify this issue.

\section{E. Temperature of the central jet}

The knowledge of the jet temperature is important to estimate the magnetic Reynolds number. An estimate of the electron temperature of the plasma in the magnetic cavity was made using time-integrated X-ray spectroscopy (photon energy of $h \nu>1 \mathrm{keV}$ ), which provided information about the hot, compressed part of the outflow. The spectrum was measured using a spherically bent mica crystal [33], which provided spatial resolution along the jet. An example of a spectrum obtained in these experiments is shown in Fig. 9a. The continuum emission $\sim 2 \mathrm{~mm}$ above the position of the foil indicates the position of a metallic diaphragm used in this experiment.

The electron temperature of the jet $T_{e}$ was estimated by considering the ratio of the combined intensity of Helium-like aluminium lines (He- $\alpha, \lambda \sim 7.76 \AA$ and $\lambda \sim 7.8$ $\AA$ ) to Hydrogen-like aluminium (Ly- $\alpha, \lambda=6.63 \AA$ ) . The dependance of these line ratios on temperature has been modeled by [34], for a cylindrical column of aluminium plasma with a diameter of $\sim 1 \mathrm{~mm}$ and an ion density of $n_{i}=10^{19} \mathrm{~cm}^{-3}$, which is close to the parameters of our experiment. It can be seen that the calculated temperature of the plasma, Fig. 9b, decreases with height, having a maximum value of $T_{e} \sim 600 \mathrm{eV}$ at $\mathrm{z} \sim 2 \mathrm{~mm}$. This plot can only provide an estimate of the temperature up to a height of $7 \mathrm{~mm}$, as the emission from the Ly- $\alpha$ line is not present at greater heights. The intensity of He$\alpha$ lines also decreases with height, and at a distance of $\sim 20 \mathrm{~mm}$ from the foil becomes non-detectable. Assuming that the disappearance of the He- $\alpha$ line is due to a decrease in temperature (and therefore also of the mean ionization state) this would require the electron temperature to drop below $T_{e} \sim 170 \mathrm{eV}$ [35]. This estimate is also 


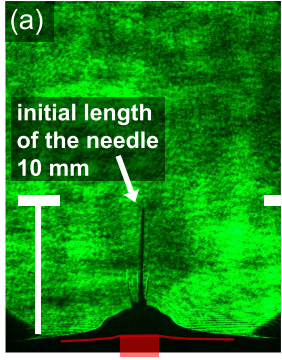

223ns

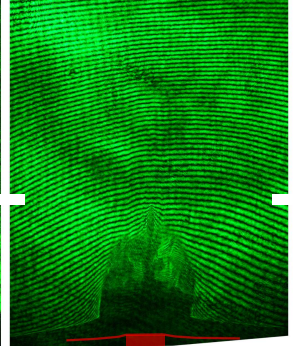

246ns

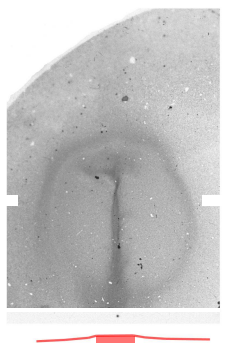

281 ns

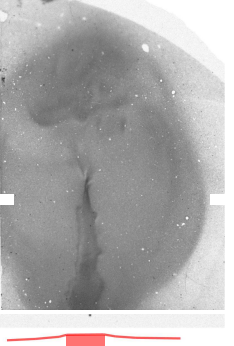

$301 n s$

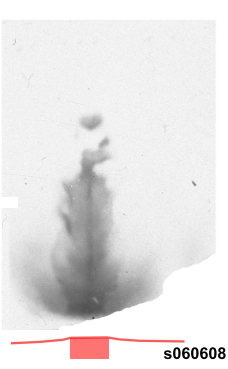

$331 \mathrm{~ns}$

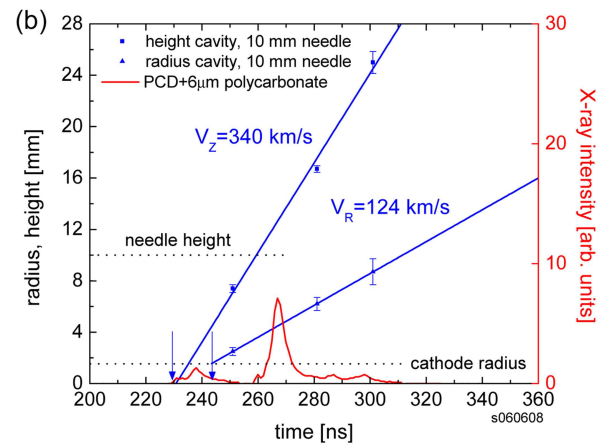

FIG. 8. (Color online) (a) Laser probing and XUV emission images of a foil with a $10 \mathrm{~mm}$ long needle on the axis obtained from the same experiment (shot s060608). (b) Measured height, radius and X-ray emission of the single magnetic cavity shown in (a).
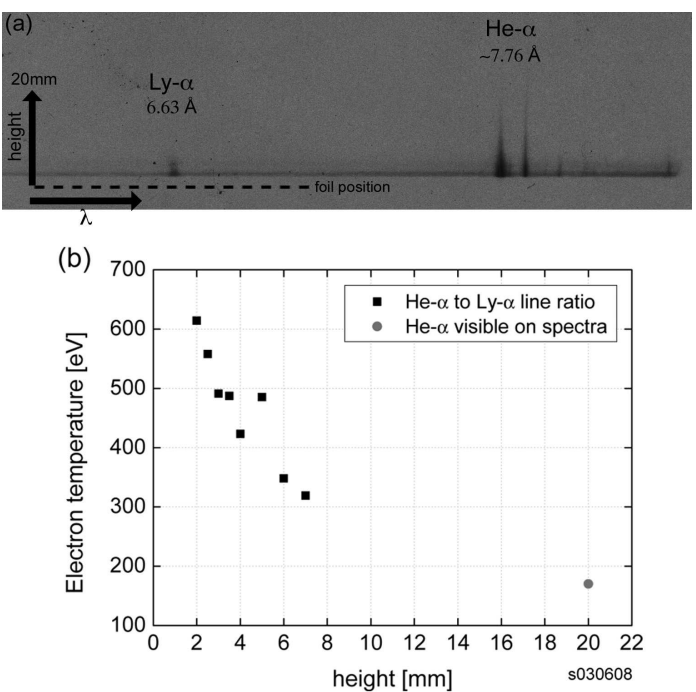

FIG. 9. (Color online) (a) Time-integrated axially-resolved $\mathrm{X}$-ray spectrum. (b) Electron temperature as a function of height obtained from the ratio of the emission lines shown in (a).

shown in Fig. 9b.

From these temperature estimates it is possible to infer characteristic values of the magnetic Reynolds number of the plasma $R e_{M}=\frac{h V}{D_{M}}$, where $h$ and $V$ are the characteristic length and velocity of the plasma, and $D_{M}$ is the magnetic diffusivity, which can be expressed as a function of the temperature $T$ and the ionization state $Z$ of the plasma [9].

For our experimental conditions we can estimate $R e_{M}$ for both the central jet and the magnetic cavity. In this estimate will use a "characteristic/average" flow velocity of $V \sim 100 \mathrm{~km} / \mathrm{s}$, noticing here that different features in the outflows have velocities in the range of $\sim 50-300 \mathrm{~km} / \mathrm{s}$. For the central jet we can consider the measured temperatures of $T=170-600 \mathrm{eV}$, jet diameter of $h \sim 1 \mathrm{~mm}$, and $Z \sim 5-10$, resulting in a range of $R e_{M} \approx 300-1000$. In the case of the magnetic cavity the plasma temperature is lower, T 50 eV according to MHD simulations of the experiment [25], but the characteristic spatial scale is larger $(h \sim 10 \mathrm{~mm})$ resulting in $R e_{M} \sim 200$. These estimates show that the magnetic Reynold numbers that characterize the plasma jet and the magnetic cavity in radial foil experiments are significantly larger than unity and thus in the regime relevant for scaled representations of astrophysical outflows.

\section{F. Trapped magnetic field inside a magnetic cavity}

The above estimates show that the magnetic Reynolds number $R e_{M}$ is much greater than unity and some magnetic flux should remain trapped inside the outflows. In particular we can expect conservation of magnetic flux accumulated in the first cavity by the time the second cavity starts to form.

The presence of toroidal magnetic field inside the expanding magnetic cavities was measured with a magnetic probe. The probe had 5 circular turns of $3 \mathrm{~mm}$ diameter 
and it was placed $10 \mathrm{~mm}$ above the foil at a radial distance of $\sim 13 \mathrm{~mm}$ from the axis, as shown in Fig. 10. The probe orientation was such that it measured the toroidal component of the magnetic field. To exclude capacitive coupling between the probe and the cathode, and also to prevent possible electron flow from the cathode region reaching the probe, a $1 \mathrm{~mm}$ thick stainless steel diaphragm was installed at $z \sim 2 \mathrm{~mm}$ above the foil. The magnetically driven jets formed through a $10 \mathrm{~mm}$ diameter aperture in the diaphragm.

An XUV emission image in Fig. 10b shows two magnetic cavities expanding above the metallic diaphragm. The position of the cathode, the diaphragm and the circular aperture on the axis are shown schematically in this figure. The position of the magnetic probe can also be seen from this image as a circular emitting boundary, due to the interaction of its outer shielding with the expanding cavity. This image shows that the addition of the probe and the diaphragm did not affect the overall dynamics of episodic jet formation (e.g. comparing with results obtained at the same time in Fig. 7a). The voltage measured by the magnetic probe is shown in Fig. 10c. It can be seen that the voltage is zero until $\sim 350 \mathrm{~ns}$, which is consistent with the time when the magnetic cavity reaches the probe as seen from imaging diagnostics.

The expected response of the magnetic probe assuming a sharp boundary for the toroidal magnetic field inside the expanding bubble can be calculated by taking into account the geometry of the probe and the dynamics of the radial expansion of the cavity, as shown in Fig. 10b. For the relatively small size of the probe we can assume that the wall of the magnetic cavity passing the probe at distance $R_{B}(t)$ from the axis is planar. When the wall of the cavity reaches the probe the trapped toroidal magnetic field inside the expanding cavity $B_{\phi}$ trap will induce a voltage in the probe from the change in the magnetic flux $\Phi$ :

$$
V_{\text {probe }}=-\frac{d \Phi}{d t}=-\frac{d}{d t}\left(B_{\phi \text { trap }}(t) S(t)\right)
$$

where $S(t)$ is the cross-section area of the probe inside the cavity, related to the radial expansion velocity of the cavity $V_{R}$.

The radial expansion velocity of the magnetic cavity for the particular shot presented in Fig. 10b was measured as $V_{R} \sim 90 \mathrm{~km} / \mathrm{s}$, thus corresponding to a signal from the magnetic probe of $B_{\phi}$ trap $\sim 0.3 \mathrm{~T}$.

This estimate can be compared with the expected magnetic field in the expanded magnetic cavity assuming that the toroidal magnetic flux present in the first cavity at the start of second cavity formation is conserved. The expected magnetic field $\left(B_{\phi} \sim 1.5 \mathrm{~T}\right)$ is of the right magnitude, though an accurate estimate of the expected field is not possible due to the uncertainty of the current loop inside the cavity at the time when it reaches the magnetic probe. To improve this comparison, spatially resolved measurements of the magnetic field inside the cavity are needed, and 3-D MHD simulations [25] indicate that the initially toroidal magnetic field becomes entangled due to the development of the kink mode of current driven instability.

\section{SUMMARY}

In this paper we have presented results from high energy density plasma experiments designed to investigate the physics of magnetically driven, supersonic, radiatively cooled plasma jets.

The most important new feature that appears in the radial foil configuration is the generation of several subsequent episodes of magnetically driven jets. The formation of the first outflow is similar to that previously observed in the radial wire array configuration. The outflow consists of a jet accelerated and confined by a toroidal magnetic field and embedded in a magnetic cavity. The cavity expansion into the surrounding ambient plasma is driven by the magnetic pressure. Reconnection of the current at the base of the cavity via a plasma expanding from the central electrode and the remnants of the foil leads to the start of the formation of the next outflow episode. The generation of several episodes is reproducible in the experiments, and up to 3-4 eruptions are observed. The formation of each episode is correlated with a burst of X-rays from the compressed/pinched jet on the axis.

The key dimensionless parameters, $P e, R e, R e_{M}$, are much greater than unity and together with the Mach number and plasma beta are all in the astrophysically appropriate regime, which makes the results of the experiments relevant to understanding the physics of launching mechanisms of astrophysical jets. The estimated values of the magnetic Reynolds number in these experiments, $R e_{M} \sim 200-1000$, are comparable or exceed those obtained in global numerical MHD simulations of astrophysical jets. The high values of the magnetic Reynolds number allowed to observe convection/trapping of the toroidal magnetic flux by the plasma outflow.

The long term evolution of jets from radial foils might help addressing questions about the spatial and temporal variability of astrophysical jets. In our experiments the formation of a "clumpy" jet is the result of the development of current-driven instabilities, which occur within the formation of the episodic ejections. The time variability in the experiments is characterized by two timescales of interest. The first is the growth time of the currentdriven MHD instabilities, of the order of a few $n s$ in the experiments. The second is the relatively longer magnetic cavity ejection period, of $\sim 30 \mathrm{~ns}$. The ratio of both timescales are in the similar regime to those observed in protostellar jets [25].

The episodic formation of magnetically driven jets observed in the experiments allows us to speculate that an episodic scenario of jet formation could be also applica- 

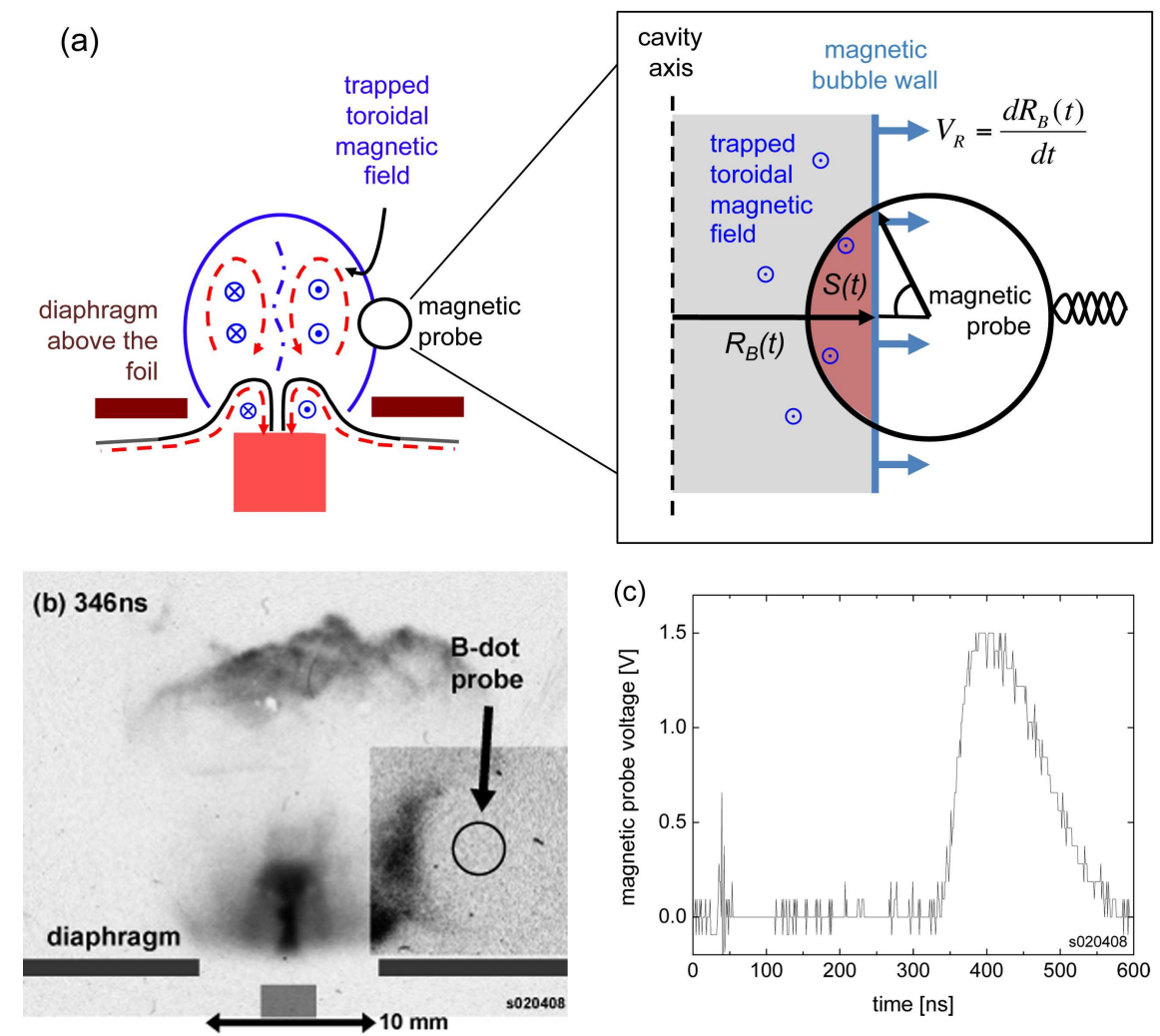

FIG. 10. (Color online) (a) Schematic setup of a radial foil with the addition of a magnetic probe and a metal diaphragm. (b) XUV emission at $346 \mathrm{~ns}$ and (c) signal from the magnetic probe. Both images were obtained from the same experiment.

ble to the formation of astrophysical jets. Indeed, steady jets confined by a toroidal magnetic field should be highly unstable to current driven instabilities, unless stabilized by e.g. a closely positioned rigid "wall" acting as a path for the return current. In the episodic jet formation scenario the time for the growth of the current driven modes is reduced to roughly the duration of one episode. The development of the instability could produce, as in the experiments, a "clumpy" outflow which still retains a high degree of collimation and propagates ballistically after the end of the episode. The resulting outflow in this scenario would have density and velocity variations due to both the current driven instability and the episodicity of the ejection. We should note that episodic jet forma- tion appeared in several numerical simulations of YSO jet launching [36-39].

\section{ACKNOWLEDGMENTS}

This work was supported by the EPSRC Grant No. EP/G001324/1, by the NNSA under DOE Cooperative Agreements No. DE-F03-02NA00057 and No. DE-SC0001063 and by a Marie Curie RTN fellowship, as part of the JETSET (Jet Simulations, Experiments and Theories) project.
[1] B. A. Remington, R. P. Drake, and D. D. Ryutov, Reviews of Modern Physics 78, 755 (2006).

[2] M. Livio, Nature 417, 125 (May 2002).

[3] M. C. Begelman, R. D. Blandford, and M. J. Rees, Reviews of Modern Physics 56, 255 (Apr. 1984).

[4] B. Balick and A. Frank, Annual Review of Astronomy and Astrophysics 40, 439 (2002).

[5] B. Reipurth, P. Hartigan, S. Heathcote, J. A. Morse, and J. Bally, The Astronomical Journal 114, 757 (Aug. 1997).

[6] B. Reipurth and J. Bally, An. Rev. Astron. and Astro- phys. 39, 403 (2001).

[7] R. D. Blandford and D. G. Payne, Monthly Notices of the Royal Astronomical Society 199, 883 (Jun. 1982).

[8] D. Ryutov, R. P. Drake, J. Kane, E. Liang, B. A. Remington, and W. M. Wood-Vasey, ApJ 518, 821 (Jun. 1999).

[9] D. D. Ryutov, R. P. Drake, and B. A. Remington, ApJS 127, 465 (Apr. 2000).

[10] J. M. Stone, N. Turner, K. Estabrook, B. Remington, D. Farley, S. G. Glendinning, and S. Glenzer, ApJS 127, 497 (Apr. 2000).

[11] L. M. Logory, P. E. Stry, and P. L. Miller, ApJS 127, 
423 (Apr. 2000).

[12] J. M. Foster, B. H. Wilde, P. A. Rosen, R. J. R. Williams, B. E. Blue, R. F. Coker, R. P. Drake, A. Frank, P. A. Keiter, A. M. Khokhlov, J. P. Knauer, and T. S. Perry, ApJL 634, L77 (Nov. 2005).

[13] P. Hartigan, J. M. Foster, B. H. Wilde, R. F. Coker, P. A. Rosen, J. F. Hansen, B. E. Blue, R. J. R. Williams, R. Carver, and A. Frank, The Astrophysical Journal 705, 1073 (Nov. 2009), arXiv:0910.0318.

[14] S. Bott, D. Haas, Y. Eshaq, U. Ueda, R. Madden, G. Collins, and F. Beg, Plasma Science, IEEE Transactions on 38, 567 (april 2010), ISSN 0093-3813.

[15] D. R. Farley, K. G. Estabrook, S. G. Glendinning, S. H. Glenzer, B. A. Remington, K. Shigemori, J. M. Stone, R. J. Wallace, G. B. Zimmerman, and J. A. Harte, Physical Review Letters 83, 1982 (Sep. 1999).

[16] K. Shigemori, R. Kodama, D. R. Farley, T. Koase, K. G. Estabrook, B. A. Remington, D. D. Ryutov, Y. Ochi, H. Azechi, J. Stone, and N. Turner, PRE 62, 8838 (Dec. 2000).

[17] S. V. Lebedev, J. P. Chittenden, F. N. Beg, S. N. Bland, A. Ciardi, D. Ampleford, S. Hughes, M. G. Haines, A. Frank, E. G. Blackman, and T. Gardiner, Astrophysical Journal 564, 113 (Jan. 2002).

[18] C. D. Gregory, J. Howe, B. Loupias, S. Myers, M. M. Notley, Y. Sakawa, A. Oya, R. Kodama, M. Koenig, and N. C. Woolsey, The Astrophysical Journal 676, 420 (Mar. 2008).

[19] S. V. Lebedev, A. Ciardi, D. J. Ampleford, S. N. Bland, S. C. Bott, J. P. Chittenden, G. N. Hall, J. Rapley, C. Jennings, M. Sherlock, A. Frank, and E. G. Blackman, Plasma Physics and Controlled Fusion 47, B465 (Dec. 2005).

[20] A. Ciardi, D. J. Ampleford, S. V. Lebedev, and C. Stehle, ApJ 678, 968 (May 2008), arXiv:0712.0959.

[21] P. Nicolaï, C. Stenz, A. Kasperczuk, T. Pisarczyk, D. Klir, L. Juha, E. Krousky, K. Masek, M. Pfeifer, K. Rohlena, J. Skala, V. Tikhonchuk, X. Ribeyre, S. Galera, G. Schurtz, J. Ullschmied, M. Kalal, J. Kravarik, P. Kubes, P. Pisarczyk, and T. Schlegel, Physics of Plasmas 15, 082701 (Aug. 2008).

[22] S. V. Lebedev, A. Ciardi, D. J. Ampleford, S. N. Bland, S. C. Bott, J. P. Chittenden, G. N. Hall, J. Rapley, C. A. Jennings, A. Frank, E. G. Blackman, and T. Lery, Monthly Notices of the Royal Astronomical Society 361, 97 (Jul. 2005), arXiv:astro-ph/0505027.

[23] A. Ciardi, S. V. Lebedev, A. Frank, E. G. Blackman, J. P. Chittenden, C. J. Jennings, D. J. Ampleford, S. N. Bland, S. C. Bott, J. Rapley, G. N. Hall, F. A. SuzukiVidal, A. Marocchino, T. Lery, and C. Stehle, Physics of
Plasmas 14, 056501 (May 2007).

[24] A. Ciardi, S. V. Lebedev, J. P. Chittenden, D. J. Ampleford, S. N. Bland, B. S. Bott, and J. Rapley, Astrophysics and Space Science 298, 277 (Jul. 2005).

[25] A. Ciardi, S. V. Lebedev, A. Frank, F. Suzuki-Vidal, G. N. Hall, S. N. Bland, A. Harvey-Thompson, E. G. Blackman, and M. Camenzind, ApJL 691, L147 (Feb. 2009), arXiv:0811.2736.

[26] F. Suzuki-Vidal, S. V. Lebedev, A. Ciardi, S. N. Bland, J. P. Chittenden, G. N. Hall, A. Harvey-Thompson, A. Marocchino, C. Ning, C. Stehle, A. Frank, E. G. Blackman, S. C. Bott, and T. Ray, Astrophysics and Space Science 322, 19 (Aug. 2009), arXiv:0904.0165.

[27] P. Gourdain, I. C. Blesener, J. B. Greenly, D. A. Hammer, P. F. Knapp, B. R. Kusse, and P. C. Schrafel, Physics of Plasmas 17, 012706 (Jan. 2010).

[28] I. H. Mitchell, J. M. Bayley, J. P. Chittenden, J. F. Worley, A. E. Dangor, M. G. Haines, and P. Choi, Review of Scientific Instruments 67, 1533 (Apr. 1996).

[29] D. D. Ryutov, M. S. Derzon, and M. K. Matzen, Reviews of Modern Physics 72, 167 (2000).

[30] M. Hipp, J. Woisetschlger, P. Reiterer, and T. Neger, Measurement 36, 53 (Jul. 2004).

[31] S. V. Lebedev, I. H. Mitchell, R. Aliaga-Rossel, S. N. Bland, J. P. Chittenden, A. E. Dangor, and M. G. Haines, Physical Review Letters 81, 4152 (Nov. 1998).

[32] B. Jones, C. A. Coverdale, C. Deeney, D. B. Sinars, E. M. Waisman, M. E. Cuneo, D. J. Ampleford, P. D. Lepell, K. R. Cochrane, J. W. Thornhill, J. P. Apruzese, A. Dasgupta, K. G. Whitney, R. W. Clark, and J. P. Chittenden, Physics of Plasmas 15, 122703 (Dec. 2008).

[33] G. N. Hall, S. A. Pikuz, T. A. Shelkovenko, S. N. Bland, S. V. Lebedev, D. J. Ampleford, J. B. A. Palmer, S. C. Bott, J. Rapley, J. P. Chittenden, and J. P. Apruzese, Physics of Plasmas 13, 082701 (Aug. 2006).

[34] J. P. Apruzese, K. G. Whitney, J. Davis, and P. C. Kepple, J. Quant. Spectrosc. Radiat. Transfer 57, 41 (1997).

[35] D. Duston and J. Davis, Physical Review A 23, 2602 (May 1981).

[36] A. P. Goodson, R. M. Winglee, and K. H. Böhm, The Astrophysical Journal 489, 199-209 (Nov. 1997).

[37] A. P. Goodson, K. H. Böhm, and R. M. Winglee, The Astrophysical Journal 524, 142-158 (Oct. 1999).

[38] A. P. Goodson, and R. M. Winglee, The Astrophysical Journal 524, 159-168 (Oct. 1999).

[39] M. M. Romanova, A. Kulkarni, M. Long, R. V. E Lovelace, J. V. Wick, G. V. Ustyugova, and A. V. Koldoba, Advances in Space Research 38, 2887-2892 (Apr. 2006). 